\title{
AN INVERSE SPECTRAL PROBLEM FOR NON-SELFADJOINT STURM-LIOUVILLE OPERATORS WITH NONSEPARATED BOUNDARY CONDITIONS
}

\author{
V. YURKO
}

\begin{abstract}
Non-selfadjoint Sturm-Liouville operators on a finite interval with nonseparated boundary conditions are studied. We establish properties of the spectral characteristics and investigate an inverse problem of recovering the operators from their spectral data. For this inverse problem we prove a uniqueness theorem and provide a procedure for constructing the solution.
\end{abstract}

\section{Introduction}

Consider the boundary value problem $L=L(q, h, \alpha, \beta, T)$ of the form

$$
\begin{aligned}
-y^{\prime \prime}+q(x) y & =\lambda y, \quad x \in[0, T], \\
y(0) & =\alpha y(T), \quad y^{\prime}(0)-h y(0)=\beta y^{\prime}(T),
\end{aligned}
$$

where $q(x) \in L(0, T)$ is a complex-valued function, $\alpha, \beta, h$ are complex numbers, and $\alpha+\beta \neq$ $0, \alpha \beta \neq 0$. We study a nonlinear inverse problem of recovering $L$ from its spectral data. For this inverse problem we prove a uniqueness theorem and provide a procedure for constructing the solution. Similar results are valid for another type of nonseparated boundary conditions: $y^{\prime}(0)-h y(0)+\alpha y(T)=y^{\prime}(T)+H y(T)-\beta y(0)=0$.

Inverse spectral problems often appear in mathematics as well as in applications [1, 2, $3,4]$. For selfadjoint Sturm-Liouville operators with separated boundary conditions, inverse spectral problems have been studied fairly completely (see the monographs $[1,2,3,4]$ and the references therein). Inverse problems for nonseparated boundary conditions in the selfadjoint case were investigated in [5]-[13] and other works. Non-selfadjoint case is more difficult for studying. Inverse problems for non-selfadjoint Sturm-Liouville operators with separated boundary conditions were investigated in $[4,14,15]$. In [16] the structure of the spectrum for the non-selfadjoint periodic case is investigated. Inverse problems for non-selfadjoint 
boundary value problem $L$ have not been studied yet. We note that the inverse problem, considered here, appears in the inverse problem theory for differential operators on spatial networks with cycles (see $[17,18,19,20,21]$ ) which have many applications in natural sciences and engineering.

Some words about the structure of the paper. In section 2 properties of the spectrum are established. The Weyl-type function and the corresponding Weyl sequence are introduced and investigated in section 3. Section 4 is devoted to the solution of the inverse spectral problem for $L$. The main result is formulated in theorem 3 . In section 5 we study the periodic boundary value problem which is a particular case of $L$. For convenience of readers, we formulate there the results from [8] (published in 1981 only in Russian) where necessary and sufficient conditions for the solvability of the inverse problem were obtained for the selfadjoint periodic case.

\section{Properties of the spetrum}

Let $C(x, \lambda), S(x, \lambda)$ and $\varphi(x, \lambda)$ be solutions of equation (1) under the initial conditions

$$
C(0, \lambda)=S^{\prime}(0, \lambda)=\varphi(0, \lambda)=1, C^{\prime}(0, \lambda)=S(0, \lambda)=0, \varphi^{\prime}(0, \lambda)=h .
$$

Clearly, $\varphi(x, \lambda)=C(x, \lambda)+h S(x, \lambda)$. Let $\lambda=\rho^{2}, \tau=\operatorname{Im} \rho, q_{1}(x)=\frac{1}{2} \int_{0}^{x} q(t) d t, q_{1}=q_{1}(T)$. It is known (see, for example [3]) that for $|\rho| \rightarrow \infty$,

$$
\begin{aligned}
& C(x, \lambda)=\cos \rho x+q_{1}(x) \frac{\sin \rho x}{\rho}+o\left(\frac{e^{|\tau| x}}{\rho}\right), \quad C^{\prime}(x, \lambda)=-\rho \sin \rho x+q_{1}(x) \cos \rho x+o\left(e^{|\tau| x}\right), \\
& S(x, \lambda)=\frac{\sin \rho x}{\rho}-q_{1}(x) \frac{\cos \rho x}{\rho^{2}}+o\left(\frac{e^{|\tau| x}}{\rho^{2}}\right), \quad S^{\prime}(x, \lambda)=\cos \rho x+q_{1}(x) \frac{\sin \rho x}{\rho}+o\left(\frac{e^{|\tau| x}}{\rho}\right) .
\end{aligned}
$$

The function $\Delta(\lambda)=\alpha \varphi(T, \lambda)+\beta S^{\prime}(T, \lambda)-(1+\alpha \beta)$ is entire in $\lambda$ of order $1 / 2$, and the set of its zeros $\Lambda=\left\{\lambda_{n}\right\}_{n \geq 0}$ (counting with multiplicities) coincides with the eigenvalues of $L$. The function $\Delta(\lambda)$ is called the characteristic function for $L$. For $|\rho| \rightarrow \infty$,

$$
\Delta(\lambda)=(\alpha+\beta)\left(\cos \rho T-\gamma+\gamma_{1} \frac{\sin \rho T}{\rho}+o\left(\frac{e^{|\tau| T}}{\rho}\right)\right),
$$

where $\gamma=\frac{\alpha \beta+1}{\alpha+\beta}$ and $\gamma_{1}=q_{1}+\frac{h \alpha}{\alpha+\beta}$. Let $\left\{\lambda_{n}^{0}\right\}_{n \geq 0}$ be the eigenvalues of $L^{0}=L(0,0, \alpha, \beta, T)$ with $q(x)=0, h=0$. Then the characteristic function of $L^{0}$ has the form

$$
\Delta^{0}(\lambda)=(\alpha+\beta)(\cos \rho T-\gamma)=\frac{\alpha+\beta}{2}\left(e^{i \rho T}+e^{-i \rho T}-2 \gamma\right)
$$

Let $z=e^{i \rho T}$, and let $z_{1,2}=\gamma \pm \sqrt{\gamma^{2}-1}$ be the roots of the equation $z^{2}-2 \gamma z+1=0$. Then $\lambda_{n}^{0}=\left(\rho_{n}^{0}\right)^{2},\left\{\rho_{n}^{0}\right\}=\left\{\rho_{n, 1}^{0}\right\} \cup\left\{\rho_{n, 2}^{0}\right\}$,

$$
\rho_{n, j}^{0}=\frac{2 n \pi}{T}-\frac{i}{T} \ln z_{j}, \quad j=1,2 .
$$


We agree that if $z=|z| e^{i \xi}, \xi \in[0,2 \pi)$ then $\ln z=\ln |z|+i \xi, \sqrt{z}=|z|^{1 / 2} e^{i \xi / 2}$.

Examples. (1) Let $\gamma=1$. Then $z_{1}=z_{2}=1, \ln z_{j}=0$,

$$
\Delta^{0}(\lambda)=(\alpha+\beta)(\cos \rho T-1), \quad \rho_{n, 1}^{0}=\frac{2 n \pi}{T}, n \geq 0, \quad \rho_{n, 2}^{0}=\frac{2 n \pi}{T}, n \geq 1 .
$$

(2) Let $\gamma=-1$. Then $z_{1}=z_{2}=-1, \ln z_{j}=\pi i, \rho_{n, 1}^{0}=\rho_{n, 2}^{0}=\frac{(2 n+1) \pi}{T}, n \geq 0$.

(3) If $\gamma \neq \pm 1$, then all eigenvalues $\left\{\lambda_{n}^{0}\right\}_{n \geq 0}$ are simple. Let, for example, $\gamma=1 / 2$. Then

$$
\rho_{n, 1}^{0}=\frac{1}{T}\left(\frac{\pi}{3}+2 n \pi\right), n \geq 0, \quad \rho_{n, 2}^{0}=\frac{1}{T}\left(-\frac{\pi}{3}+2 n \pi\right), n \geq 1 .
$$

If $\gamma=0$, then $z_{1,2}= \pm i, \ln z_{1}=\pi i / 2, \ln z_{2}=3 \pi i / 2, \rho_{n}^{0}=(n+1 / 2) \frac{\pi}{T}, n \geq 0$.

Using (3), by the known method (see [22]) one gets $\lambda_{n}=\rho_{n}^{2},\left\{\rho_{n}\right\}=\left\{\rho_{n, 1}\right\} \cup\left\{\rho_{n, 2}\right\}$,

$$
\rho_{n, j}=\rho_{n, j}^{0}+\varepsilon_{n, j}, \quad \varepsilon_{n, j}=o(1), \quad n \rightarrow \infty .
$$

More precisely (see [23]),

$$
\begin{aligned}
& \varepsilon_{n, j}=\frac{\gamma_{1}}{2 \pi n}+o\left(\frac{1}{n}\right), \quad \text { if } \gamma \neq \pm 1, \\
& \varepsilon_{n, j}=\frac{1}{2 \pi n}\left(\gamma_{1}+(-1)^{j} h / 2\right)+o\left(\frac{1}{n}\right), \quad \text { if } \gamma= \pm 1, \alpha=\beta, \\
& \varepsilon_{n, j}=o\left(\frac{1}{\sqrt{n}}\right), \quad \text { if } \gamma= \pm 1, \alpha \neq \beta .
\end{aligned}
$$

Theorem 1. (i) Let $\gamma \neq 1$ (i.e. $\Delta^{0}(0) \neq 0$ ). Then

$$
\Delta(\lambda)=(\alpha+\beta-1-\alpha \beta) \prod_{n=0}^{\infty} \frac{\lambda_{n}-\lambda}{\lambda_{n}^{0}}, \quad \lambda_{n}^{0} \neq 0 .
$$

(ii) Let $\gamma=1$ (i.e. $\Delta^{0}(0)=0$ ). Then

$$
\Delta(\lambda)=-\frac{(\alpha+\beta) T^{2}}{2}\left(\lambda-\lambda_{0}\right) \prod_{n=1}^{\infty} \frac{\lambda_{n}-\lambda}{\lambda_{n}^{0}}, \quad \lambda_{2 n}^{0}=\lambda_{2 n-1}^{0}=\left(\frac{2 \pi n}{T}\right)^{2} .
$$

Proof. It follows from (4) that

$$
\dot{\Delta}^{0}(\lambda)=-(\alpha+\beta) T \frac{\sin \rho T}{2 \rho}, \quad \dot{\Delta}^{0}(\lambda):=\frac{d}{d \lambda} \Delta^{0}(\lambda)=\frac{1}{2 \rho} \frac{d}{d \rho} \Delta^{0}(\lambda),
$$

and consequently, $\Delta^{0}(0)=\alpha+\beta-1-\alpha \beta, \dot{\Delta}^{0}(0)=-(\alpha+\beta) T^{2} / 2 \neq 0$. Using Hadamard's factorization theorem we get

$$
\Delta^{0}(\lambda)=A^{0} \prod_{n=0}^{\infty} \frac{\lambda_{n}^{0}-\lambda}{\lambda_{n}^{0}}, A^{0}=\alpha+\beta-1-\alpha \beta, \lambda_{n}^{0} \neq 0, \quad \text { if } \gamma \neq 1
$$




$$
\Delta^{0}(\lambda)=B^{0} \lambda \prod_{n=1}^{\infty} \frac{\lambda_{n}^{0}-\lambda}{\lambda_{n}^{0}}, B^{0}=-\frac{(\alpha+\beta) T^{2}}{2}, \lambda_{2 n}^{0}=\lambda_{2 n-1}^{0}=\left(\frac{2 \pi n}{T}\right)^{2}, \quad \text { if } \quad \gamma=1 .
$$

Let for definiteness, $\gamma \neq 1, \Delta(0) \neq 0$ (other cases are treated similarly). Then, by Hadamard's factorization theorem,

$$
\Delta(\lambda)=A \prod_{n=0}^{\infty} \frac{\lambda_{n}-\lambda}{\lambda_{n}}
$$

where $A \neq 0$ is a constant. Therefore,

$$
\frac{\Delta(\lambda)}{\Delta^{0}(\lambda)}=\frac{A}{A^{0}} \prod_{n=0}^{\infty} \frac{\lambda_{n}^{0}}{\lambda_{n}} \prod_{n=0}^{\infty} \frac{\lambda-\lambda_{n}}{\lambda-\lambda_{n}^{0}}=\frac{A}{A^{0}} \prod_{n=0}^{\infty} \frac{\lambda_{n}^{0}}{\lambda_{n}} \prod_{n=0}^{\infty}\left(1-\frac{\lambda_{n}-\lambda_{n}^{0}}{\lambda-\lambda_{n}^{0}}\right) .
$$

By virtue of (3)-(5),

$$
\lim _{\lambda \rightarrow-\infty} \frac{\Delta(\lambda)}{\Delta^{0}(\lambda)}=1, \quad \lim _{\lambda \rightarrow-\infty} \prod_{n=0}^{\infty}\left(1-\frac{\lambda_{n}-\lambda_{n}^{0}}{\lambda-\lambda_{n}^{0}}\right)=1
$$

This yields

$$
A=A^{0} \prod_{n=0}^{\infty} \frac{\lambda_{n}}{\lambda_{n}^{0}}
$$

and we arrive at (6). Relation (7) is proved similarly.

\section{The Weyl sequence}

Denote $d(\lambda):=S(T, \lambda)$. Zeros $\mathcal{V}=\left\{v_{n}\right\}_{n \geq 1}$ of $d(\lambda)$ (counting with multiplicities) coincide with the eigenvalues of the boundary value problem $L_{0}$ for equation (1) with Dirichlet boundary conditions $y(0)=y(T)=0$. The function $d(\lambda)$ is called the characteristic function for $L_{0}$. Let $G_{\delta}:=\{\rho:|\rho-(n \pi) / T| \geq \delta \forall n\}$. Then (see [1], [3])

$$
\begin{aligned}
\sqrt{v_{n}} & =\frac{n \pi}{T}+\frac{q_{1}}{n \pi}+o\left(\frac{1}{n}\right), \quad n \rightarrow \infty, \\
d(\lambda) & =\frac{T^{3}}{\pi^{2}} \prod_{n=1}^{\infty} \frac{v_{n}-\lambda}{n^{2}}, \\
|d(\lambda)| & \geq C|\rho|^{-1} e^{|\tau| T}, \quad \rho \in G_{\delta}, \quad|\rho| \geq \rho^{*} .
\end{aligned}
$$

Let $\Phi(x, \lambda)$ be the solution of equation (1) under the conditions $\Phi(0, \lambda)=1, \Phi(T, \lambda)=0$. Denote $M(\lambda):=\Phi^{\prime}(0, \lambda)$. The function $M(\lambda)$ is called the Weyl function for $L_{0}$. Clearly,

$$
\begin{aligned}
M(\lambda) & =-\frac{d_{1}(\lambda)}{d(\lambda)}, \\
\Phi(x, \lambda) & =C(x, \lambda)+M(\lambda) S(x, \lambda),
\end{aligned}
$$

where $d_{1}(\lambda):=C(T, \lambda)$. Relation (12) is used for solving inverse spectral problems for the boundary value problem $L_{0}$ by the method of spectral mappings $[4,14,15]$. Since $d_{1}(\lambda)=$ $O\left(e^{|\tau| T}\right)$, it follows from (10)-(11) that

$$
|M(\lambda)| \leq C|\rho|, \quad \rho \in G_{\delta},|\rho|>\rho^{*} .
$$


For $|\rho| \rightarrow \infty$, one has

$$
\begin{aligned}
& d_{1}(\lambda)=\cos \rho T+q_{1} \frac{\sin \rho T}{\rho}+o\left(\frac{e^{|\tau| T}}{\rho}\right), \quad d(\lambda)=\frac{\sin \rho T}{\rho}-q_{1} \frac{\cos \rho T}{\rho^{2}}+o\left(\frac{e^{|\tau| T}}{\rho}\right), \\
& \dot{d}(\lambda)=\frac{1}{2 \rho}\left(\frac{T \cos \rho T}{\rho}+\frac{\left(q_{1} T-1\right) \sin \rho T}{\rho}+o\left(\frac{e^{|\tau| T}}{\rho}\right)\right), \quad \dot{d}(\lambda):=\frac{d}{d \lambda} d(\lambda) .
\end{aligned}
$$

In particular, this yields

$$
M(\lambda)=i \rho+o(1), \quad|\rho| \rightarrow \infty, \arg \rho \in[\delta, \pi-\delta],
$$

Let $m_{n}$ be the multiplicity of $v_{n}\left(v_{n}=v_{n+1}=\ldots=v_{n+m_{n}-1}\right)$. Denote

$$
S:=\left\{n \geq 2: v_{n-1} \neq v_{n}\right\} \cup\{1\}, \quad I:=\left\{n \in S: m_{n}>1\right\} .
$$

It follows from (8) that $I$ is a finite set, i.e. $m_{n}=1$ for sufficiently large $n\left(n>n^{*}\right)$. Fix $n \in S$. Using (11) we obtain that in a neighborhood of the point $\lambda=v_{n}$ the function $M(\lambda)$ has the representation

$$
M(\lambda)=\sum_{v=0}^{m_{n}-1} \frac{M_{n+v}}{\left(\lambda-v_{n}\right)^{v+1}}+M_{n}^{*}(\lambda),
$$

where $M_{n}^{*}(\lambda)$ is regular in $\lambda=v_{n}$, and the coefficients $M_{n+v}, v=\overline{0, m_{n}-1}$ are calculated from $d_{1}^{(v)}\left(v_{n}\right)$ and $d^{\left(v+m_{n}\right)}\left(v_{n}\right)$ for $v=\overline{0, m_{n}-1}$. More precisely,

$$
\begin{aligned}
M_{n+m_{n}-1-v} & =-\frac{1}{d_{0 n}^{0}}\left(d_{v n}^{1}+\sum_{k=0}^{v-1} M_{n+m_{n}-1-k} d_{v-k, n}^{0}\right), \quad v=\overline{0, m_{n}-1}, \\
d_{v n}^{1} & :=\frac{1}{v !} d_{1}^{(v)}\left(v_{n}\right), \quad d_{v n}^{0}:=\frac{1}{\left(v+m_{n}\right) !} d^{\left(v+m_{n}\right)}\left(v_{n}\right), \quad v=\overline{0, m_{n}-1} .
\end{aligned}
$$

In particular, if $m_{n}=1$ (i.e. $n \in S \backslash I$ ), then

$$
M_{n}=-\frac{d_{1}\left(v_{n}\right)}{\dot{d}\left(v_{n}\right)} .
$$

The sequence $\left\{M_{n}\right\}_{n \geq 1}$ is called the Weyl sequence, and the data $\mathscr{D}:=\left\{v_{n}, M_{n}\right\}_{n \geq 1}$ are called the spectral data. One has

$$
\dot{d}\left(v_{n}\right)=\frac{(-1)^{n} T}{2 v_{n}}\left(1+o\left(\frac{1}{n}\right)\right), \quad d_{1}\left(v_{n}\right)=(-1)^{n}\left(1+o\left(\frac{1}{n}\right)\right), \quad n \rightarrow \infty .
$$

Using (8), (16) and (17) we get

$$
M_{n}=-\frac{2 n^{2} \pi^{2}}{T^{3}}\left(1+o\left(\frac{1}{n}\right)\right), \quad n \rightarrow \infty .
$$

Take $\theta>0$ such that $d( \pm i \theta) \neq 0$. For $n \in S$, we denote

$$
b_{v, n}:=\frac{1}{v !}\left(\frac{z}{z^{2}+\theta^{2}}\right)_{\mid z=v_{n}}^{(v)}, \quad v=\overline{0, m_{n}-1}
$$


Let us consider the function

$$
N(\lambda):=\sum_{n \in S} \sum_{v=0}^{m_{n}-1}\left(\frac{1}{\left(\lambda+v_{n}\right)^{v+1}}+b_{v, n}\right) M_{n+v} .
$$

By virtue of (8) and (18),

$$
\left(\frac{1}{\lambda-v_{n}}+\frac{v_{n}}{v_{n}^{2}+\theta^{2}}\right) M_{n}=\left(\frac{\theta^{2}+\lambda v_{n}}{\left(\lambda-v_{n}\right)\left(v_{n}^{2}+\theta^{2}\right)}\right) M_{n}=O\left(\frac{1}{n^{2}}\right), \quad n \rightarrow \infty,
$$

and consequently, the series in (19) converges absolutely and uniformly in $\lambda$ on compacts without $\left\{v_{n}\right\}$.

Theorem 2. The specification of the spectral data $\mathscr{D}$ uniquely determines the Weyl function by the formula

$$
M(\lambda)=N(\lambda)+a
$$

where $N(\lambda)$ is defined by (19), and

$$
a=\lim _{\lambda \rightarrow-\infty}(-N(\lambda)+i \rho)
$$

Proof. Consider the functions

$$
J_{N}(\lambda):=\frac{1}{2 \pi i} \int_{\Gamma_{N}}\left(\frac{1}{\lambda-z}+\frac{z}{z^{2}+\theta^{2}}\right) M(z) d z,
$$

where $\Gamma_{N}=\left\{z:|z|=\frac{\pi^{2}}{T^{2}}(N+1 / 2)^{2}\right\}$. By virtue of (13),

$$
\left(\frac{1}{\lambda-z}+\frac{z}{z^{2}+\theta^{2}}\right) M(z)=O\left(\frac{1}{z^{3 / 2}}\right)
$$

Therefore, the integral in (22) converges absolutely and uniformly in $\lambda$ on compacts without $\Gamma_{N}$, and

$$
\lim _{N \rightarrow \infty} J_{N}(\lambda)=0
$$

On the other hand one can calculate $J_{N}(\lambda)$, using the residue theorem. This yelds

$$
\begin{aligned}
J_{N}(\lambda) & =-M(\lambda)+\sum_{n \in S, v_{n} \in i n t \Gamma_{N}} \operatorname{Res}_{z=v_{n}}\left(\frac{1}{\lambda-z}+\frac{z}{z^{2}+\theta^{2}}\right) M(z)+a, \\
a & =\operatorname{Res}_{z=i \theta} \frac{z}{z^{2}+\theta^{2}} M(z)+\operatorname{Res}_{z=-i \theta} \frac{z}{z^{2}+\theta^{2}} M(z) .
\end{aligned}
$$

By virtue of (15), one has

$$
\operatorname{Res}_{z=v_{n}} \frac{M(z)}{\lambda-z}=\sum_{v=0}^{m_{n}-1} \frac{M_{n+v}}{\left(\lambda-v_{n}\right)^{v+1}}, \quad \operatorname{Res}_{z=v_{n}} \frac{z M(z)}{z^{2}+\theta^{2}}=\sum_{v=0}^{m_{n}-1} b_{v, n} M_{n+v} .
$$


Taking (23) and (14) into account we arrive at (20) and (21).

\section{Inverse problems}

In this section we formulate and study an inverse problem of recovering $L$ from its spectral data. The main result is Theorem 3, where an algorithm for the solution of the inverse problem is provided, and the uniqueness of the solution is proved. Denote

$$
D(\lambda)=\alpha \varphi(T, \lambda)+\beta S^{\prime}(T, \lambda), \quad Q(\lambda)=\alpha \varphi(T, \lambda)-\beta S^{\prime}(T, \lambda) .
$$

Then

$$
\varphi(T, \lambda)=\frac{1}{2 \alpha}(D(\lambda)+Q(\lambda)), \quad S^{\prime}(T, \lambda)=\frac{1}{2 \beta}(D(\lambda)-Q(\lambda)) .
$$

Since $\varphi(x, \lambda) S^{\prime}(x, \lambda)-\varphi^{\prime}(x, \lambda) S(x, \lambda) \equiv 1$, it follows that

$$
Q^{2}(\lambda)=D^{2}(\lambda)-4 \alpha \beta\left(1+\varphi^{\prime}(T, \lambda) S(T, \lambda)\right)
$$

and consequently,

$$
\dot{Q}(\lambda) Q(\lambda)=\dot{D}(\lambda) D(\lambda)-2 \alpha \beta\left(\dot{\varphi}^{\prime}(T, \lambda) S(T, \lambda)+\varphi^{\prime}(T, \lambda) \dot{S}(T, \lambda)\right)
$$

Let $n \in S$. Denote

$$
\omega_{n}=\left\{\begin{array}{l}
0, \quad Q\left(v_{n}\right)=0, \\
+1, Q\left(v_{n}\right) \neq 0, \arg Q\left(v_{n}\right) \in[0, \pi), \\
-1, Q\left(v_{n}\right) \neq 0, \arg Q\left(v_{n}\right) \in[\pi, 2 \pi),
\end{array}\right.
$$

$\omega_{n v}:=d_{1}^{(v)}\left(v_{n}\right), v=\overline{0, m_{n}-1}, I_{0}=\left\{n \in I: \omega_{n}=0\right\}, I_{1}=\left\{n \in I: \omega_{n} \neq 0\right\}$. The sequence $\Omega=$ $\left\{\omega_{n}\right\}_{n \in S} \cup\left\{\omega_{n v}\right\}_{n \in I_{0}, v=\overline{1, m_{n}-1}}$ is called the $\Omega$ - sequence for $L$. We note that if $I=\varnothing$ (i.e. $m_{n}=1$ for all $n)$, then $\Omega=\left\{\omega_{n}\right\}_{n \geq 1}$. For example, $I=\varnothing$, if $q(x), h, \alpha, \beta$ are real, i.e. in the self-adjoint case. Let $\alpha$ and $\beta$ be known a priori and fixed. The inverse problem is formulated as follows.

Inverse problem 1. Given $\Lambda, \mathcal{V}$ and $\Omega$, construct $q(x), h$ and $T$.

Obviously, in general it is not possible to recover uniquely the coefficients $\alpha$ and $\beta$, but it is possible to calculate $\gamma$. Note that Inverse problem 1 is a generalization of the classical inverse problems in the selfadjoint case (see [5]-[13]).

The solution of Inverse problem 1 can be constructed as follows. Using (8) we calculate $T$. By virtue of (6)-(7) and (9) we find $d(\lambda)$ and $\Delta(\lambda)$. Then we calculate $D(\lambda)=\Delta(\lambda)+(1+\alpha \beta)$. It follows from (24) that $Q^{2}\left(v_{n}\right)=D^{2}\left(v_{n}\right)-4 \alpha \beta, n \in S$, and consequently,

$$
Q\left(v_{n}\right)=\omega_{n} \sqrt{D^{2}\left(v_{n}\right)-4 \alpha \beta}, \quad n \in S .
$$


Since $\varphi\left(T, v_{n}\right)=C\left(T, v_{n}\right)$, we construct

$$
\omega_{n 0}=\frac{1}{2 \alpha}\left(D\left(v_{n}\right)+Q\left(v_{n}\right)\right), \quad n \in S .
$$

Now we are going to calculate the Weyl sequence $\left\{M_{n}\right\}_{n \geq 1}$.

Case 1. Let $n \in S, n \notin I$ (i.e. $m_{n}=1$ ). Taking (16) into account we get

$$
M_{n}=\frac{\omega_{n 0}}{\dot{d}\left(v_{n}\right)} .
$$

Case 2. Let $n \in I_{1}$ (i.e. $m_{n}>1, \omega_{n} \neq 0$ ). Then it follows from (25) that

$$
(\dot{Q}(\lambda) Q(\lambda))_{\mid \lambda=v_{n}}^{(v-1)}=(\dot{D}(\lambda) D(\lambda))_{\mid \lambda=v_{n}}^{(v-1)}, \quad v=\overline{1, m_{n}-1}
$$

Using (29) we find $Q^{(v)}\left(v_{n}\right), v=\overline{1, m_{n}-1}$. Since $d_{1}(\lambda)=\frac{1}{2 \alpha}(D(\lambda)+Q(\lambda))-h d(\lambda)$, we construct $\omega_{n v}, v=\overline{1, m_{n}-1}$ by the formula

$$
\omega_{n v}=\frac{1}{2 \alpha}\left(D^{(v)}\left(v_{n}\right)+Q^{(v)}\left(v_{n}\right)\right), \quad v=\overline{1, m_{n}-1} .
$$

Case 3. Let $n \in I_{0}$ (i.e. $m_{n}>1, \omega_{n}=0$ ). Then $\omega_{n v}, v=\overline{1, m_{n}-1}$ are given a priori.

Thus, we have constructed the Weyl sequence $\left\{M_{n}\right\}_{n \geq 1}$. It is also possible to construct the Weyl function $M(\lambda)$ by (20)-(21). Our calculations show that Inverse problem 1 is reduced to the following inverse problem.

Inverse problem 2. Given $\mathscr{D}=\left\{v_{n}, M_{n}\right\}_{n \geq 1}$, construct $q(x)$.

This non-selfadjoint inverse problem was solved in [15]. For this inverse problem the uniqueness theorem was proved and a constructive procedure for its solution was provided in [15]. Moreover, necessary and sufficient conditions for the solvability of Inverse problem 2 were also established. We note that for the selfadjoint case Inverse problem 2 is equivalent to the classical inverse Sturm-Liouville problem (see [1, 2, 3, 4]).

Thus, we have obtained a procedure for the solution on Inverse problem 1 and proved its uniqueness, i.e. the following theorem holds.

Theorem 3. The specification of $\Lambda, \mathcal{V}$ and $\Omega$ uniquely determines $q(x), h$ and $T$. The solution of Inverse problem 1 can be found by the following algorithm.

Algorithm 1. Given $\Lambda, \mathcal{V}$ and $\Omega$.

(1) Find T, using (8).

(2) Construct $\Delta(\lambda)$ and $d(\lambda)$ by (6) -(7) and (9).

(3) Calculate $D(\lambda)=\Delta(\lambda)+(1+\alpha \beta)$. 
(4) Find $Q\left(v_{n}\right), n \in S$, by (26).

(5) Calculate $\omega_{n 0}, n \in S$, by (27).

(6) Construct $Q^{(v)}\left(v_{n}\right), n \in I_{1}, v=\overline{1, m_{n}-1}$ using (29).

(7) Find $\omega_{n v}, n \in I_{1}, v=\overline{1, m_{n}-1}$, via (30).

(8) Calculate the Weyl sequence $\left\{M_{n}\right\}_{n \geq 1}$ using (28) and the recurrent formula

$$
M_{n+m_{n}-1-v}=-\frac{1}{d_{0 n}^{0}}\left(d_{v n}^{1}+\sum_{k=0}^{v-1} M_{n+m_{n}-1-k} d_{v-k, n}^{0}\right), \quad n \in S, v=\overline{0, m_{n}-1}, d_{v n}^{1}:=\frac{1}{v !} \omega_{n v} .
$$

(9) Find the potential $q(x)$ by solving Inverse problem 2 (see [15]).

(10) Calculate the coefficient $h$, using (3).

\section{Periodic boundary conditions}

In this section we study the periodic boundary value problem which is a particular case of L. For convenience of readers, we formulate here the results from [8] (published in 1981 only in Russian) where necessary and sufficient conditions for the solvability of the inverse problem were obtained for the selfadjoint periodic case. In [5] one can find another conditions for the characterization of the periodic spectrum.

Let us consider the periodic boundary value problem $L^{\prime}:=L(q(x), 0,1,1, \pi)$ with $\alpha=\beta=$ $1, h=0, T=\pi$, and real-valued potential $q(x) \in L_{2}(0, \pi)$. Denote

$$
p(\lambda)=1-\frac{1}{2}\left(C(\pi, \lambda)+S^{\prime}(\pi, \lambda)\right) .
$$

The zeros $\Lambda=\left\{\lambda_{n}\right\}_{n \geq 0}$ of $p(\lambda)$ coincide with the eigenvalues of $L^{\prime}$. It is known (see [1]) that $\lambda_{n}$ are real, and

$$
\lambda_{2 n-1}=(2 n)^{2}+a+\kappa_{2 n-1}^{\prime}, \lambda_{2 n}=(2 n)^{2}+a+\kappa_{2 n}^{\prime},\left\{\kappa_{n}^{\prime}\right\} \in l_{2}, \lambda_{n} \leq \lambda_{n+1}, \lambda_{n}<\lambda_{n+2},
$$

where $a=\frac{1}{\pi} \int_{0}^{\pi} q(t) d t$. Moreover, it follows form (7) that

$$
p(\lambda)=\frac{\pi^{2}}{2}\left(\lambda-\lambda_{0}\right) \prod_{n=1}^{\infty} \frac{\lambda_{n}-\lambda}{\lambda_{n}^{0}}, \quad \lambda_{2 n-1}^{0}=\lambda_{2 n}^{0}=(2 n)^{2} .
$$

Denote $s(\lambda)=p(\lambda)-2$. The zeros $\left\{\mu_{n}\right\}_{n \geq 1}$ of $s(\lambda)$ coincide with the eigenvalues of the antiperiodic boundary value problem $L^{\prime \prime}:=L(q(x), 0,-1,-1, \pi)$. It is known that $\mu_{n}$ are real, $\lambda_{0}<$ $\mu_{1} \leq \mu_{2}<\lambda_{1} \leq \lambda_{2}<\ldots$, and

$$
\mu_{2 n-1}=(2 n-1)^{2}+a+\kappa_{2 n-1}^{\prime \prime}, \quad \mu_{2 n}=(2 n-1)^{2}+a+\kappa_{2 n}^{\prime \prime}, \quad\left\{\kappa_{n}^{\prime \prime}\right\} \in l_{2} .
$$

Moreover,

$$
\max _{\lambda \in\left[\lambda_{2 n}, \lambda_{2 n+1}\right]} p(\lambda) \geq 2
$$


We will show that condition (33) is also sufficient for the solvability of the inverse problem. It is known (see $[1,2,3,4]$ ) that the zeros $\left\{v_{n}\right\}_{n \geq 1}$ of $d(\lambda):=S(\pi, \lambda)$ are real, and

$$
v_{n}=n^{2}+a+\kappa_{n}^{0}, \quad\left\{\kappa_{n}^{0}\right\} \in l_{2}, \quad v_{2 n} \in\left[\lambda_{2 n-1}, \lambda_{2 n}\right], \quad v_{2 n-1} \in\left[\mu_{2 n-1}, \mu_{2 n}\right] .
$$

Denote $Q(\lambda)=C(\pi, \lambda)-S^{\prime}(\pi, \lambda), \Omega^{\prime}=\left\{\omega_{n}\right\}_{n \geq 1}$, where $\omega_{n}=\operatorname{sign} Q\left(v_{n}\right)$, i.e. $\omega_{n}=0$, if $Q\left(v_{n}\right)=0$, and $\omega_{n}= \pm 1$, if $\pm Q\left(v_{n}\right)>0$. The sequence $\Omega^{\prime}$ is called the sign sequence for $q$.

Now we will give the characterization of the spectrum of $L^{\prime}$. We will say that a real sequence $\left\{\lambda_{n}\right\}_{n \geq 0} \in \mathscr{S}$ if there exists $L^{\prime}$ such that $\left\{\lambda_{n}\right\}_{n \geq 0}$ is the spectrum of $L^{\prime}$. Denote by $J$ the set of sequences $\left\{\omega_{n}\right\}_{n \geq 1}$ such that $\omega_{n}=0$ if $p\left(v_{n}\right) s\left(v_{n}\right)=0$, and $\omega_{n}= \pm 1$ if $p\left(v_{n}\right) s\left(v_{n}\right) \neq 0$.

Theorem 4. Let the real numbers $\left\{\lambda_{n}\right\}_{n \geq 0}$ of the form (31) be given. For $\left\{\lambda_{n}\right\}_{n \geq 0} \in \mathscr{S}$ it is necessary and sufficient that (33) holds, where $p(\lambda)$ is constructed by (32). Moreover, if we additionally have real numbers $\left\{v_{n}\right\}_{n \geq 1}$ satisfying (34), where $\left\{\mu_{n}\right\}_{n \geq 1}$ are zeros of $s(\lambda)=p(\lambda)-2$, and the sequence $\left\{\omega_{n}\right\}_{n \geq 1} \in J$, then there exists a unique real function $q(x) \in L_{2}(0, \pi)$ for which $\left\{\lambda_{n}\right\}_{n \geq 0}$ and $\left\{v_{n}\right\}_{n \geq 1}$ are the eigenvalues of $L^{\prime}$ and $L_{0}$, respectively, and $\left\{\omega_{n}\right\}_{n \geq 1}$ is the sign sequence for $q$.

\section{Acknowledgement}

This research was supported in part by DAAD and grants 10-01-00099 and 10-01-92001NSC of Russian Foundation for Basic Research and Taiwan National Science Council.

\section{References}

[1] V. A. Marchenko, Sturm-Liouville operators and their applications "Naukova Dumka", Kiev, 1977; English transl., Birkhäuser, 1986.

[2] B. M. Levitan, Inverse Sturm-Liouville problems. Nauka, Moscow, 1984; English transl., VNU Sci.Press, Utrecht, 1987.

[3] G. Freiling and V. A. Yurko, Inverse Sturm-Liouville Problems and their Applications. NOVA Science Publishers, New York, 2001.

[4] V. A. Yurko, Method of Spectral Mappings in the Inverse Problem Theory, Inverse and Ill-posed Problems Series. VSP, Utrecht, 2002.

[5] V. A. Marchenko and I. V. Ostrovskii, A characterization of the spectrum of the Hill operator, Math.USSR-Sb. 26 (1975), 4, 493-554.

[6] O. A. Plaksina, Inverse problems of spectral analysis for the Sturm-Liouville operators with nonseparated boundary conditions, Math. USSR-Sb. 59(1988), 1, 1-23.

[7] V. A. Yurko, An inverse problem for second order differential operators with regular boundary conditions Math. Notes 18 (1975), 3-4, 928-932.

[8] V. A. Yurko, On an periodic problem, Diff. Equations and Theory of Functions, (Russian), Saratov Univ., Saratov, 1981, 109-115.

[9] V. A. Yurko, On differential operators with nonseparated boundary conditions, Funct. Anal. and Appl 28(1994), 295-297. 
[10] V. A. Yurko, The inverse spectral problem for differential operators with nonseparated boundary conditions, J. Math. Anal. Appl. 250(2000), 266-289.

[11] G. Freiling and V. A. Yurko, On the stability of constructing a potential in the central symmetry case, Applicable Analysis 90(2011), 1819-1828.

[12] M. Gasymov, I. M. Guseinov and I. M. Nabiev, An inverse problem for the Sturm-Liouville operator with nonseparated self-adjoint boundary conditions, Sib. Mat. Zh. 31(1990), 46-54; English transl. in Siberian Math. J. 31, no.6 (1990), 910-918.

[13] I. M. Guseinov and I. M. Nabiev, Solution of a class of inverse Sturm-Liouville boundary value problems, Mat. Sb. 186(1995), 35-48; English transl.in Sb. Math. 186(1995), 661-674.

[14] S. A. Buterin, On inverse spectral problems for non-selfadjoint Sturm-Liouville operator on a finite interval, Journal of Math. Analysis and Appl. 335 (2007), 739-749.

[15] S. A. Buterin, Inverse problems for non-self-adjoint Sturm-Liouville operators, Schriftenreiche des Fachbereichs Mathematik, SM-DU-745, Universitaet Duisburg-Essen, 2012.

[16] V. A. Tkachenko and J.-J. Sansuc, Characterization of the periodic and anti-periodic spectra of nonselfadjoint Hill's operators, Operator Theory, Advances and Appl. 98, 216-224, Birkhaeuser, Basel, 1997.

[17] V. A. Yurko, Inverse problems for Sturm-Liouville operators on graphs with a cycle, Operators and Matrices, 2(2008), 543-553.

[18] V. A. Yurko, Recovering Sturm-Liouville operators from spectra on a graph with a cycle, Matem. Sbornik 200(2009), 147-160; English transl. in Sbornik: Mathematics 200(2009), 1403-1415.

[19] V.A. Yurko, On an inverse spectral problem for differential operators on a hedgehog-type graph, Doklady Mathematics 79(2009), 250-254.

[20] V. A. Yurko, Inverse problems for Sturm-Liouville operators on bush-type graphs, Inverse Problems 25(2009), 105008, 14pp.

[21] V. A. Yurko, Inverse spectral problems for differential operators on arbitrary compact graphs, Journal of Inverse and Ill-Posed Proplems 18(2010), 245-261.

[22] M. A. Naimark, Linear Differential Operators, 2nd ed., "Nauka", Moscow, 1969; English transl. of 1st ed., Parts I, II, Ungar, New York, 1967, 1968.

[23] V. A. Yurko, Recovering non-selfadjoint Sturm-Liouville operators with nonseparated boundary conditions, Schriftenreiche des Fachbereichs Mathematik, SM-DU-746, Universitaet Duisburg-Essen, 2012, 11pp.

Department of Mathematics, Saratov University, Astrakhanskaya 83, Saratov 410012, Russia.

E-mail: yurkova@info.sgu.ru 